\title{
Messungen zum Einfluss von Wasser auf die Beladungserkennung von Dieselpartikelfiltern mit Mikrowellentechnik
}

\author{
Markus Feulner, Andreas Müller, Ralf Stöber, Gerhard Fischerauer, Ralf Moos \\ Bayreuth Engine Research Center (BERC), Universität Bayreuth, \\ Universitätsstr. 30, 95447 Bayreuth, Germany \\ funktionsmaterialien@uni-bayreuth.de
}

\begin{abstract}
Zusammenfassung:
Dieselpartikelfilter (DPF) sind im Zuge immer strengerer Abgasgrenzwerte mittlerweile unverzichtbarer Bestandteil eines effizienten Abgasnachbehandlungssystems geworden. Dabei spielt die Kenntnis der Rußbeladung eines Filters im Betrieb eine wichtige Rolle. Eine neuartige Mikrowellen-gestützte Methode vermag berührungslos, direkt und in situ die Beladung des Filters mit Ruß zu bestimmen. Die grundsätzliche Eignung dieser Methode für Anwendungen im Realabgas ist bereits nachgewiesen, nun sollen typische Störeinflüsse wie der Einfluss von an den Ruß angelagerter Feuchte bei verschiedenen Temperaturen auf die Signalmerkmale Resonanzfrequenz und Transmission dieser hochfrequenzgestützten Beladungserkennung beleuchtet werden. Dazu wurde ein Filter am Prüfstandsmotor definiert mit Ruß beladen, ausgebaut, gewogen und anschließend in einem Klimaschrank unterschiedlichen Temperaturen und Luftfeuchten ausgesetzt. Es konnte ein merklicher Einfluss der Luftfeuchte als Funktion der Temperatur auf beide Signalmerkmale beobachtet werden, wobei die Abhängigkeit von der Luftfeuchte mit steigender Temperatur zunimmt. Insgesamt sind die Störeinflüsse zwar gering im Vergleich zu denen, die durch eine Änderung der Rußbeladung hervorgerufen werden, sie sind jedoch bei einer späteren Anwendung trotzdem zu berücksichtigen und soweit möglich zu korrigieren.
\end{abstract}

Key words: Dieselpartikelfilter (DPF), Mikrowellen, Cavity Perturbation, Beladungserkennung

\section{Einleitung}

Um die immer strenger werdenden Abgasgrenzwerte für hochmoderne dieselbetriebene Verbrennungskraftmaschinen, einzuhalten, sind Dieselpartikelfilter (DPF) heute unverzichtbarer Bestandteil eines jeden Abgasnachbehandlungssystems. Von Zeit zu Zeit muss der Filter jedoch durch einen Abbrand des Rußes regeneriert werden, was durch Eingriffe in die Motorsteuerung während des Betriebs initiiert wird. Für eine effiziente Regenerationsstrategie ist dabei die genaue Kenntnis der Rußbeladung des Filters notwendig. Stand der Technik hierzu ist derzeit eine modellgestützte Beladungserkennung in Verbindung mit der Messung des Differenzdruckes über dem DPF [1]. Eine neuartige Mikrowellen-gestützte Methode kann die Filterbeladung direkt, berührungslos und in situ messen [2]. Die prinzipielle Eignung des Systems zur Beladungserkennung wurde bereits mehrfach im Realabgas nachgewiesen $[3,4,5]$. In dieser Arbeit sollen nun verschiedene Einflussparameter auf die Signalmerkmale, die typischerweise im
Realbetrieb auftreten können, in einem Klimaschrank systematisch untersucht werden.

\section{Messprinzip}

Für die mikrowellengestützte Beladungserkennung wird ausgenutzt, dass sich der Filtermonolith in einem metallischen Gehäuse (Canning) befindet. Dieses fungiert aufgrund der guten elektrischen Leitfähigkeit des Metalls als Resonator. Werden mit Stiftkopplern elektromagnetische Wellen in das Gehäuse eingekoppelt, formen sich im Inneren stehende Wellen (Resonanzen) bei charakteristischen Frequenzen [2]. Misst man nun die Reflexion der Wellen an der gleichen Stelle an denen sie eingekoppelt werden $\left(\left|S_{11}\right|, \mid S_{22} I\right)$, bzw. die Transmission durch den Filter $\left(\left|S_{12}\right|,\left|S_{21}\right|\right)$, kann man diese Resonanzfrequenzen als Lage von Minima in den Reflexionsspektren und als Lage von Maxima in den Transmissionsspektren ablesen. Die Spektren sind dabei abhängig von der Resonatorgeometrie und von den elektrischen Eigenschaften des Dielektrikums im Inneren (el. Leitfähigkeit, Permittivität). Lagert sich im Betrieb Dieselruß 
im Filter an, der wesentlich leitfähiger ist als das keramische Filtermaterial, so ändert sich die Form der Reflexions- und Transmissionsspektren [2]. Allerdings beeinflussen auch andere Parameter, wie z.B. die Feuchte und die Temperatur, die aus den Spektren abgeleiteten Signalmerkmale.

Neben der Verschiebung der Resonanzfrequenz einer ausgewählten Resonanzmode soll hier auch der Mittelwert des Transmissionsspektrums in einem breiten Bereich von 0,8 - 2,5 GHz betrachtet werden.

\section{Versuchsaufbau und -durchführung}

Ein kommerzieller keramischer Wall-Flow-Filter (Aluminiumtitanat, unbeschichtet, $300 \mathrm{cpsi}$, 5,66 “ $\times 6$ 6", $\left.V_{D P F}=2,47 \mathrm{I}\right) \quad$ wird in ein Edelstahlgehäuse montiert (Fig. 1). Vor und nach dem Filterelement ragt je ein Stiftkoppler zum Einkoppeln und Messen der elektromagnetischen Wellen in das Gehäuse. An den beiden offenen Enden des Cannings sind Drahtgitter angebracht, um die Resonatorlänge eindeutig zu definieren. Durch zwei Konen wird der Durchmesser auf die Größe des Abgasrohres reduziert.

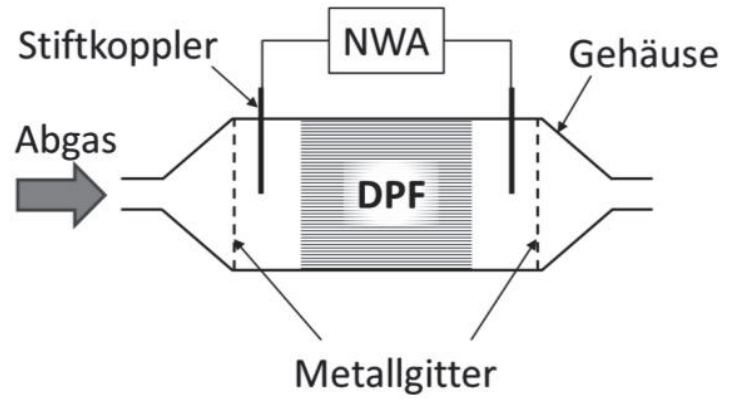

Fig. 1. Schematischer Aufbau der Messanordnung.

Der Filter wird am Motorprüfstand (3 I TDIMotor, 6 Zyl.) mit einer Rußmenge von $1,5 \mathrm{~g} / \mathrm{I}_{\mathrm{DPF}}$ beladen. Dabei wird der Motor während der gesamten Beladungsdauer (ca. $100 \mathrm{~min}$ ) mit einer konstanten Drehzahl von $2350 \mathrm{~min}^{-1}$ und $20 \%$ Last betrieben. Die eingespeicherte Rußmenge wird durch Wiegen des Filters vor und nach dem Versuch bestimmt. Nach der Beladung wird das gesamte Filtergehäuse aus dem Abgasstrang ausgebaut und in einem Klimaschrank unterschiedlichen Luftfeuchten bei verschiedenen Temperaturen ausgesetzt. Anders als im Abgasstrang wird der Filter hier nicht direkt durchströmt. Es werden lediglich die Umgebungsbedingungen (Temperatur, $\vartheta$, und relative Feuchte, r.F.) eingestellt und protokolliert. Vorne und hinten, an den Enden der Konen, ist das Gehäuse jedoch offen, wodurch ein Gasaustausch möglich ist.
Mit einem Netzwerkanalysator (NWA, Anritsu MS2028B) werden sowohl während der Beladung am Motorprüfstand als auch im Klimaschrank kontinuierlich Reflexions- und Transmissionsspektren in einem Frequenzbereich zwischen 0,5 und $2,5 \mathrm{GHz}$ aufgenommen.

\section{Messung während der Filterbeladung}

Figur 2 zeigt zwei beispielhafte Signalmerkmale während der Rußbeladung im stationären Motorbetrieb eines zu Beginn völlig rußfreien DPF auf 1,5 $\mathrm{g}_{\mathrm{Ru}} / \mathrm{l}_{\mathrm{DPF}}$ : oben die Frequenz $f_{\text {res }}$ einer ausgewählten Resonanzmode (TE ${ }_{112^{-}}$ Mode) und unten den im Bereich zwischen 0,8 $2,5 \mathrm{GHz}$ gemittelten Betrag des Transmissionsparameters (IS $\quad$ I $)$. Mit zunehmender Beladung des Filters mit Ruß sinken sowohl die Resonanzfrequenz als auch die gemittelte Transmission. Dabei verlaufen beide Kurven linear. Dieses Verhalten ist auf die Änderung der elektrischen Leitfähigkeit und Permittivität bei Rußanlagerung zurückzuführen und steht in Übereinstimmung mit früheren Ergebnissen und der Literatur [2, 3, 4]. Dadurch, dass die Motorparameter während des Beladens nicht verändert werden, wirken hier noch keine Störeinflüsse, wie z.B. Temperaturänderung (konstante Abgastemperatur $\vartheta_{\text {Abgas }} \approx 230{ }^{\circ} \mathrm{C}$ ). Insgesamt ist in der Resonanzfrequenz eine Änderung von ca. 18 $\mathrm{MHz}$ pro $\mathrm{g}_{\text {Ruß }} / \mathrm{l}_{\text {DPF }}$ (bzw. 1,5\% pro $\mathrm{g}_{\mathrm{Ru \beta}} / \mathrm{l}_{\mathrm{DPF}}$ ) zu beobachten. Der Wert der gemittelten Transmission verringert sich um $1,5 \mathrm{~dB}$ pro $g_{\text {Ruß }} / I_{\text {DPF. }}$. Der Einfluss von Temperatur und Feuchte soll nun gesondert bei konstanter Rußmenge im Filter untersucht werden.

Die Schwankungen zu Beginn der Messung entstehen während des Anschaltens des Motors und Einstellens der Betriebsparameter.

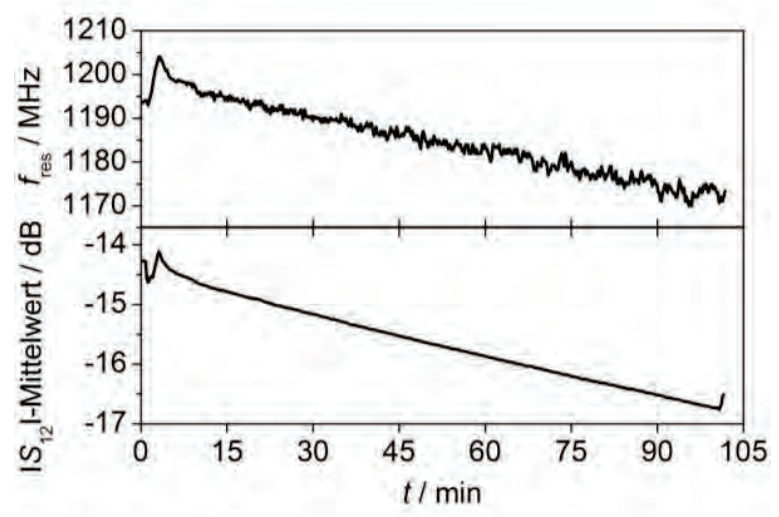

Fig. 2. Beispielhafte Resonanzfrequenz (oben) und gemittelter Transmissionsfaktor (unten) während der Rußbeladung auf 1,5 g/IDPF (2350 U/min, $20 \%$ Last, $\vartheta_{\text {Abgas }} \approx 230^{\circ} \mathrm{C}$ ). 


\section{Messung der Feuchteabhängigkeit}

Die Ergebnisse der Messung im Klimaschrank sind im Folgenden zusammengefasst. Es werden relative Luftfeuchten von 13, 50 und $80 \%$ eingestellt und dabei jeweils die Temperatur in drei Stufen variiert. Bei $50{ }^{\circ} \mathrm{C}$ wird zusätzlich auch eine relative Luftfeuchte von $2 \%$ eingestellt. Figur 3 zeigt, wie sich die Frequenz der Resonanzmode bei ca. $1190 \mathrm{MHz}$ mit der Umgebungstemperatur verändert. Es ist die gleiche Resonanzmode wie in Figur 2 während der Beladung dargestellt. Der gemittelte Wert des simultan aufgenommenen Transmissionsspektrums wird in Figur 4 gezeigt. Der Filter wird zuvor jeweils ca. zwei Stunden lang den konstanten Umgebungsbedingungen ausgesetzt, so dass davon ausgegangen werden kann, dass der DPF tatsächlich die Temperatur der Umgebung angenommen hat und ein ausreichender Gasaustausch möglich war.

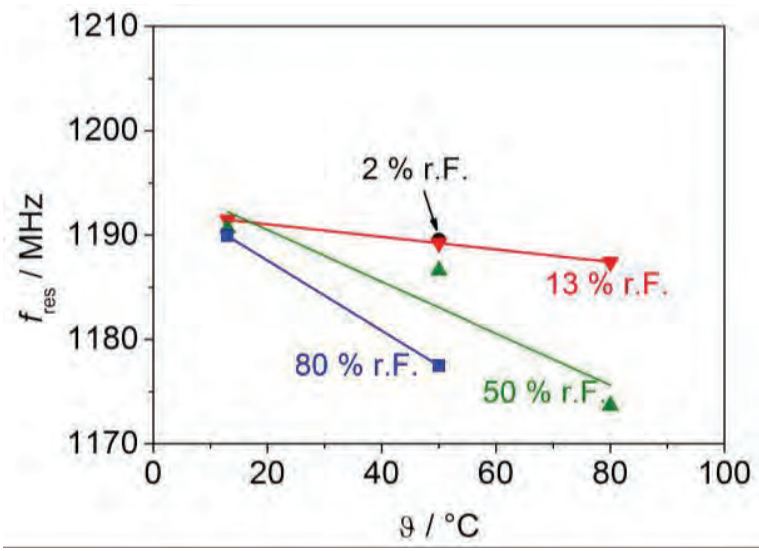

Fig. 3. Resonanzfrequenz ( $\left.f_{\text {res }}\right)$ bei unterschiedlicher Luftfeuchte über der Umgebungstemperatur.

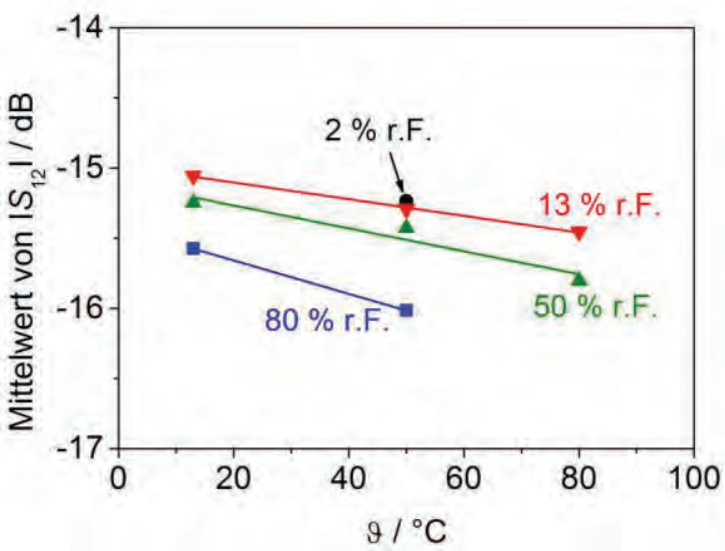

Fig. 4. Gemittelter $\left|S_{12}\right|$-Wert bei unterschiedlicher Luftfeuchte über der Umgebungstemperatur.

Es ist eine deutliche Abhängigkeit beider Signalmerkmale von der Temperatur und der Luftfeuchte zu erkennen. Sowohl eine Erhöhung der Temperatur, als auch eine höhere Feuchtigkeit bewirken dabei eine
Verringerung der Resonanzfrequenz und des gemittelten Transmissionswertes. Grund dafür könnte sein, dass Wasser neben einer Änderung der Permittivität im Filter auch eine Erhöhung der Leitfähigkeit bewirkt. Den gleichen Effekt hat auch eine Temperaturerhöhung.

Dabei nimmt der Temperatureinfluss mit steigender Luftfeuchte deutlich zu. Dieses Verhalten ist bei der Resonanzfrequenz wesentlich stärker ausgeprägt als beim Mittelwert des $\left|S_{12}\right|$-Spektrums. Dies erscheint auch plausibel, da die Resonanzfrequenz die Eigenschaft eines einzelnen Resonatormodus darstellt, der Mittelwert der Transmissionsdämpfung dagegen durch Überlagerung zahlreicher, teils gegenläufiger Prozesse entsteht.

Die gemessenen Werte liegen alle über denen des beladenen Filters am Ende der Beladungsmessung. Dies lässt sich mit der vergleichsweise niedrigen Temperatur in der Klimakammer erklären.

Zur Veranschaulichung sind in Figur 5 die Werte der Signalmerkmale Resonanzfrequenz und gemittelter Betrag des Transmissionsfaktors über der relativen Feuchte bei einer konstanten Umgebungstemperatur von $50{ }^{\circ} \mathrm{C}$ aufgetragen. Die Abhängigkeit der beiden Signalmerkmale von der Luftfeuchte ist dabei gleichartig und nicht linear, ließe sich jedoch in einer realen Anwendung des Systems z.B. mit Kennfeldern gut korrigieren.

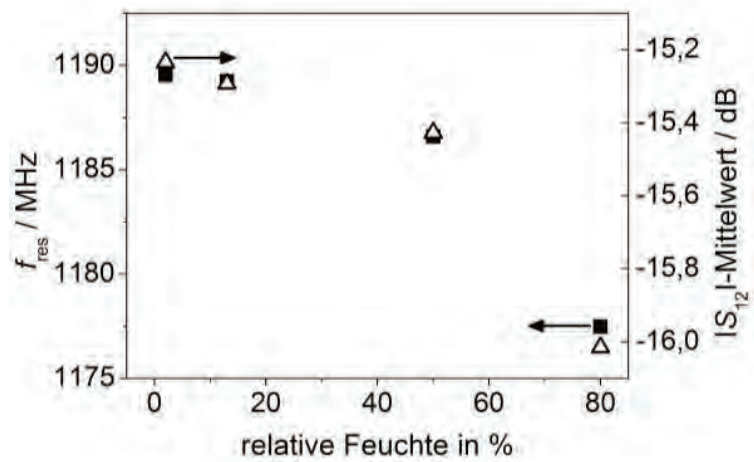

Fig. 5. Die Signalmerkmale Resonanzfrequenz (ם) und gemittelter Betrag des Transmissionsfaktors $(\Delta)$ über der Luftfeuchte bei einer Umgebungstemperatur von $50{ }^{\circ} \mathrm{C}$.

\section{Ergebniszusammenfassung und Ausblick}

Es ist ein deutlicher Einfluss sowohl der Temperatur als auch der Luftfeuchte erkennbar. Dabei nimmt der Temperatureinfluss mit steigender Luftfeuchte zu. Eine Erhöhung beider Größen spiegelt eine Erhöhung der Rußbeladung vor. So führt beispielsweise bei $80{ }^{\circ} \mathrm{C}$ eine Erhöhung der Luftfeuchte von $15 \%$ 
auf $\quad 50 \% \quad z u \quad$ einer Änderung der Resonanzfrequenz von $15 \mathrm{MHz}$, was dem Effekt von fast $1 \mathrm{~g}_{\mathrm{RuB}} / \mathrm{I}_{\mathrm{DPF}}$ entspricht. Das Transmissionssignal ist im Vergleich dazu wesentlich unempfindlicher gegenüber der Feuchte. Hier wirkt sich ebenfalls eine Erhöhung der Luftfeuchte von $15 \%$ auf $50 \%$ bei $80{ }^{\circ} \mathrm{C}$ auf den Messwert mit einer Änderung von nur 0,38 dB aus, wohingegen $1 \mathrm{~g}$ Ruß $/_{\mathrm{DPF}}$ eine Signaländerung von 1,5 dB hervorruft. Der durch die Rußbeladung hervorgerufene Effekt überwiegt also deutlich. Trotzdem darf dieser Störeinfluss bei einer Anwendung nicht vernachlässigt werden und ist zu korrigieren. Gerade eine Temperaturmessung ist im Abgasstrang relativ einfach möglich. Man könnte sogar einen der beiden Stiftkoppler als Thermoelement ausführen, wie dies in [6] beschrieben wurde. Es bleibt zu untersuchen, ob die Resonanzfrequenzen einzelner Resonanzmoden unempfindlich gegen eine Änderung der Rußbeladung sind und nur auf die hier untersuchten Störeinflüsse reagieren. Beispielsweise könnte man sich Resonanzmoden vorstellen, deren Frequenz vor allem von der Geometrie und kaum vom Filtermaterial beeinflusst wird. Diese könnten dann sogar ohne weitere Sensoren zur Korrektur des Beladungssignals hinzugezogen werden. Außerdem werden weitere Messungen hinsichtlich des Temperatureinflusses und des Feuchteeinflusses, insbesondere bei höheren Temperaturen, folgen.

\section{Danksagung}

Diese Arbeit wird von der Deutschen Forschungsgemeinschaft (DFG) unter den Förderkennzeichen MO 1060/6-2 und FI 956/3-2 unterstützt.

\section{Literatur}

[1] D. Rose, T. Boger, Different Approaches to Soot Estimation as Key Requirement for DPF Applications, SAE Technical Paper 2009-01-1262 (2009), doi: 10.4271/2009-01-1262

[2] G. Fischerauer, M. Förster, R. Moos, Sensing the soot load in automotive diesel particulate filters by microwave methods, Measurement Science and Technology 21, 035108 (2010), doi: 10.1088/0957-0233/21/3/035108

[3] M. Feulner, G. Hagen, A. Piontkowski, A. Müller, G. Fischerauer, D. Brüggemann, R. Moos, InOperation Monitoring of the Soot Load of Diesel Particulate Filters: Initial Tests, Topics in Catalysis, 56, 483-488 (2013), doi: $10.1007 / \mathrm{s} 11244-013-0002-9$

[4] A. Sappok, J. Parks II, V. Prikhodko, Loading and Regeneration Analysis of a Diesel Particulate Filter with a Radio Frequency-Based Sensor,
SAE Technical Paper 2010-01-2126 (2010), doi: $10.4271 / 2010-01-2126$

[5] R. Moos, G. Beulertz, S. Reiß, G. Hagen, G. Fischerauer, M. Votsmeier, J. Gieshoff, Overview: Status of the microwave-based automotive catalyst state diagnosis, Topics in Catalysis, 56, 358-364 (2013), doi: $10.1007 / s 11244-013-9980-x$

[6] G. Fischerauer, A. Gollwitzer, G. Hagen, R. Moos, M. Spörl, M. Wedemann, Vorrichtung und Verfahren zur Steuerung eines

Abgasnachbehandlungssystems, das einen Abgaskatalysator beinhaltet, Deutsche Offenlegungsschrift DE 102008 012050, 2008. 